\title{
Chemical Structure of Surfactant-Grafted Polyacrylamide Used in Oilfields
}

\author{
Tingting Jiang1, Haiyu Wang2, Baohui Wang1 \\ ${ }^{1}$ Institute of New Energy Chemistry and Environmental Science, College of Chemistry \& Chemical Engineering, \\ Northeast Petroleum University, Daqing, China \\ ${ }^{2}$ First Oil Plant, Daqing Oilfield Company Limited, Daqing, China \\ Email: jiangtingting82@163.com
}

Received 4 June 2014; revised 21 July 2014; accepted 3 August 2014

Copyright (C) 2014 by authors and Scientific Research Publishing Inc.

This work is licensed under the Creative Commons Attribution International License (CC BY).

http://creativecommons.org/licenses/by/4.0/

(c) (i) Open Access

\begin{abstract}
Surfactant-grafted Polyacrylamide (S-PAM) flooding is a new technique used in Daqiang Oil Field in China and also a novel way for improving the recovery efficiency of primary oil layer after polymer flooding. S-PAM flooding technology is perfected gradually with the proceeding of multiple polymer surfactant oil displacement tests. Test results showed that the S-PAM used in field was greatly different with conventional ones. Therefore, it is necessary to study the chemical structure of S-PAM. Aiming at making clear the chemical structure of S-PAM, this study characterized the two commonly used S-PAM in oil field through chemical analysis and Fourier infrared spectrometer detection and analyzed the main composition and chemical structure of the two S-PAM.
\end{abstract}

\section{Keywords}

Surfactant-Grafted Polyacrylamide, Chemical Structure, Oilfield

\section{Introduction}

Relevant data reveal that Surfactant-grafted Polyacrylamide (S-PAM) has a framework constituted by ordinary polyacrylamide hydrocarbon chains. By grafting and copolymerizing the functional monomers and functional group to the lateral groups of the molecular chains, the original polymer hydrocarbon chains can be converted into the hydrocarbon chains containing a large amount of surfactants. Therefore, the aqueous solution of S-PAM is provided with the physical and chemical properties of the polymer and surfactant, as well as the new properties which are absent in the two agents. Generally, surfactant reduces the interfacial tension between oil and water in oil-water two-phase system to improve oil displacement efficiency. The S-PAM disperses the nonpolar substances in non-water system (including crude oil, coal power, cement, pigment, and so on) into emulsion and 
thus overcomes the application limitations of conventional surfactants in non-aqueous system [1] [2].

As for unhydrous oil, the S-PAM can unify the phase viscosity, phase activity, thickening (water) ability, and solubilizing (oil) ability and reduced the tension of oil-water interface significantly in the aqueous solution with low molecular weight and concentration. The chemical agent of single component formed by special molecular design is called as multivariate graft polymer surfactants. Since the molecules contains hydrophilic and lipophilic groups (also known as hydrophobic group), such surfactants are known as parent molecule.

The molecular structures of polymer surfactants include random form, blocking form, and grafting form etc. This study investigated the Surfactant-grafted Polyacrylamide. Grafting surfactant is a kind of functional polymer compound with clear structure and also shows certain special effects in aspect of surface activity. According to the differences of the hydrophilic radicals in the molecules, the S-PAM can be generally classified into cationic surfactant, anionic surfactant, nonionic surfactant, and amphoteric surfactants. In broad sense, there are also surfactants composed of cationic and nonionic surfactants and those composed of anionic and nonionic surfactants.

The application performance of S-PAM greatly depends on the molecular structure and application condition. The molecular structure therein is the most fundamental factor and also the primary study aim for developing functional, efficient and new products. The molecular structure of S-PAM mainly includes the chemical composition of chains, the sequence distribution of chain structure, and the iconicity, charge density, and geometric structure (linear, branched, and cross linked) of molecular chains etc. It is of great significance of determining and characterizing the chemical structure of S-PAM for grasping the relationship between the chemical structure and performance of S-PAM, selecting S-PAM, optimizing usage mode and usage condition, improving synthesis methods and polymerization condition, as well as developing new S-PAM [3]-[8].

This study analyzed the composition and structure of Lianhua S-PAM and Haibo S-PAM using chemical analysis method (chemical surfactant group identification method) and instrument analysis method (infrared spectroscopy) etc.

\section{Experimental}

\subsection{Materials and Instruments}

Two origins of S-PAM were used in this experiment: Daqing Refining \& Chemical Company S-PAM (Lianhua type III), which is called Lianhua S-PAM ;Shanghai Haibo company S-PAM (Haibo type III), which is called Haibo S-PAM; polymer: polyacrylamide (the Fourth Oil Plant of Daqing Oilfield Company Limited, at molecular weight of 35,000,000); surfactants: cetyl trimethyl ammonium bromide(CTAB) (cationic surfacant), petroleum sulfonate surfactants (anionic surfactant, the Fourth Oil Production Plant); Main instrument: Btuker Tensor27 infrared spectroscopy.

\subsection{Methods}

The structure and composition of the S-PAM were firstly studied using chemical analysis method to analyze the surfactant types in the two S-PAM on whole and accurately determine the group type in the following infrared spectrum test.

Determination of cationic surfactants: we took a certain volume of S-PAM solution and adjust the $\mathrm{pH}$ value to 7. Then we added $10 \mathrm{ml}$ bromophenol blue reagent into the solution. In case of cationic surfactant, the solution shows blue color.

Determination of anionic and nonionic surfactants: we took a certain volume of S-PAM solution and added 10 $\mathrm{mL}$ methylene blue solution and $5 \mathrm{~mL}$ chloroform into the solution. The solution obtained was violently oscillated for 2 - $3 \mathrm{~s}$ and then stayed for layering. The colors of the two layers were observed. In case of cationic surfactant, the chloroform layer was blue. Then we added surfactant solution again and repeated the operations above. The chloroform layer then turned dark blue. In case of nonionic surfactants, the solution was milky solution or the two layers present the same color.

To further determine the group types in the surfactants produced by Daqing and Shanghai, instruments were used for analysis. In S-PAM analysis field, infrared spectra were mainly used for quantitative analysis, especially for the quantitative analysis of the functional groups of S-PAM. According to the characteristic absorption of compounds, it was aware of the functional groups in the compounds, which were conductive to determine and 
verify the types of related compounds. This section used the infrared spectrum analysis method to analyze the compositions of the two surfactants.

\section{Results and Discussion}

\subsection{The Compositions and Structure of S-PAM}

\subsubsection{Determination of Cationic Surfactant}

The solutions in Figure 1 are distilled water blank control, Lianhua S-PAM, Haibo S-PAM, polyacrylamide (used by the Fourth Oil Plant of Daqing Oilfield Company Limited, at molecular weight of 35,000,000), CTAB (cationic surfactant) in turn from left to right.

The experiments preliminarily showed that there was no cationic surfactant involved in above two S-PAM.

\subsubsection{Determination of Anionic and Nonionic Surfactants}

As shown in Figure 2, the solutions are distilled water blank control, Lianhua S-PAM, Haibo S-PAM, polyacrylamide (used by the Fourth Oil Plant of Daqing Oilfield Company Limited, at molecular weight of $35,000,000$ ), CTAB (cationic surfactant) in turn from left to right.

As shown in this figure, Lianhua S-PAM contains nonionic surfactant and possibly contains cationic surfactant; Haibo S-PAM contains both nonionic surfactant and cationic surfactant.

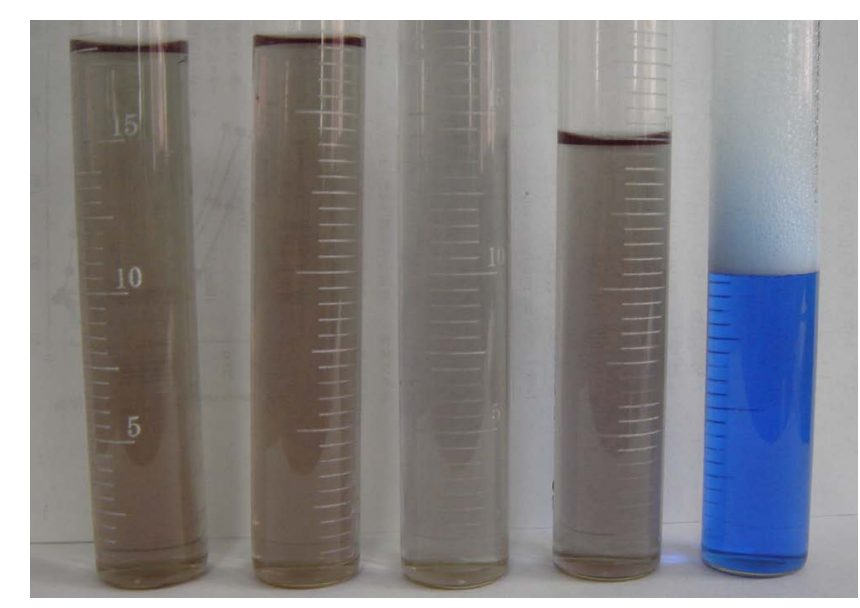

Figure 1. Determination of cationic surfactant.

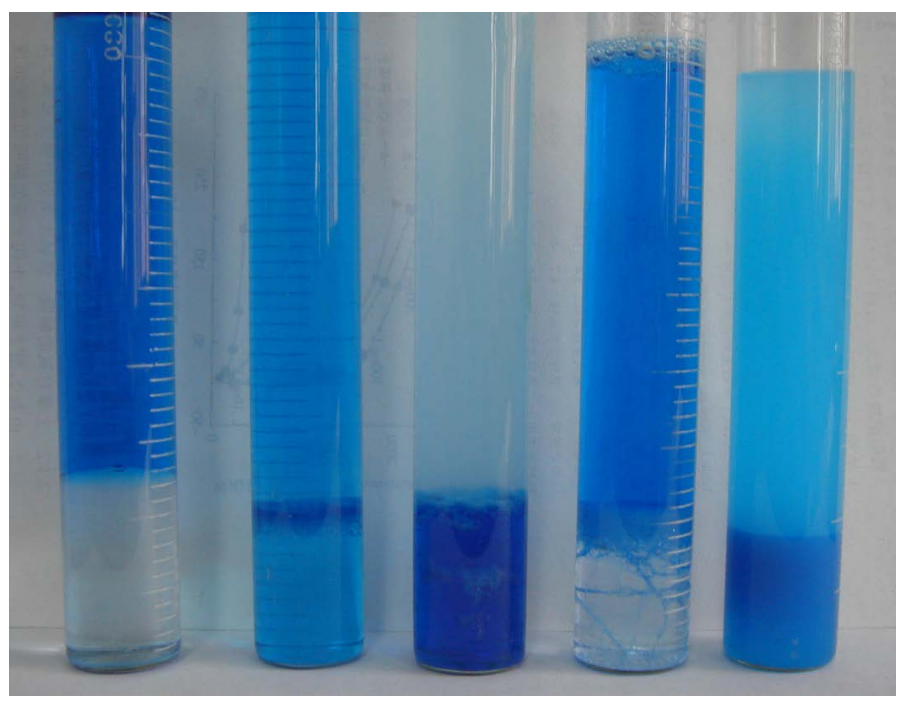

Figure 2. Determination of anionic and nonionic surfactants. 


\subsubsection{Identification of the Anions in S-PAM Solution}

The anions were primarily examined to obtain the grafting groups in S-PAM solution. The primary examination results showed that the anions included sulfate radical ions $\left(\mathrm{SO}_{4}^{2-}\right)$ and chloride ions $\mathrm{Cl}^{-}$. The barium chloride and silver nitrate at certain concentrations were mixed with S-PAM solutions at certain concentration. The reaction phenomena were observed. In case of white precipitate and the precipitate that were insoluble after the hydrochloric acid and barium chloride were added in, it was proved that there were $\mathrm{SO}_{4}^{2-}$ and $\mathrm{Cl}^{-}$. Table 1 shows the test results:

\subsubsection{Infrared Characterization}

The compositions of the two S-PAM were analyzed using infrared spectrum in this section. Figure 3 and Figure 4 show the infrared spectra of the two S-PAM (Table 2 and Table 3).

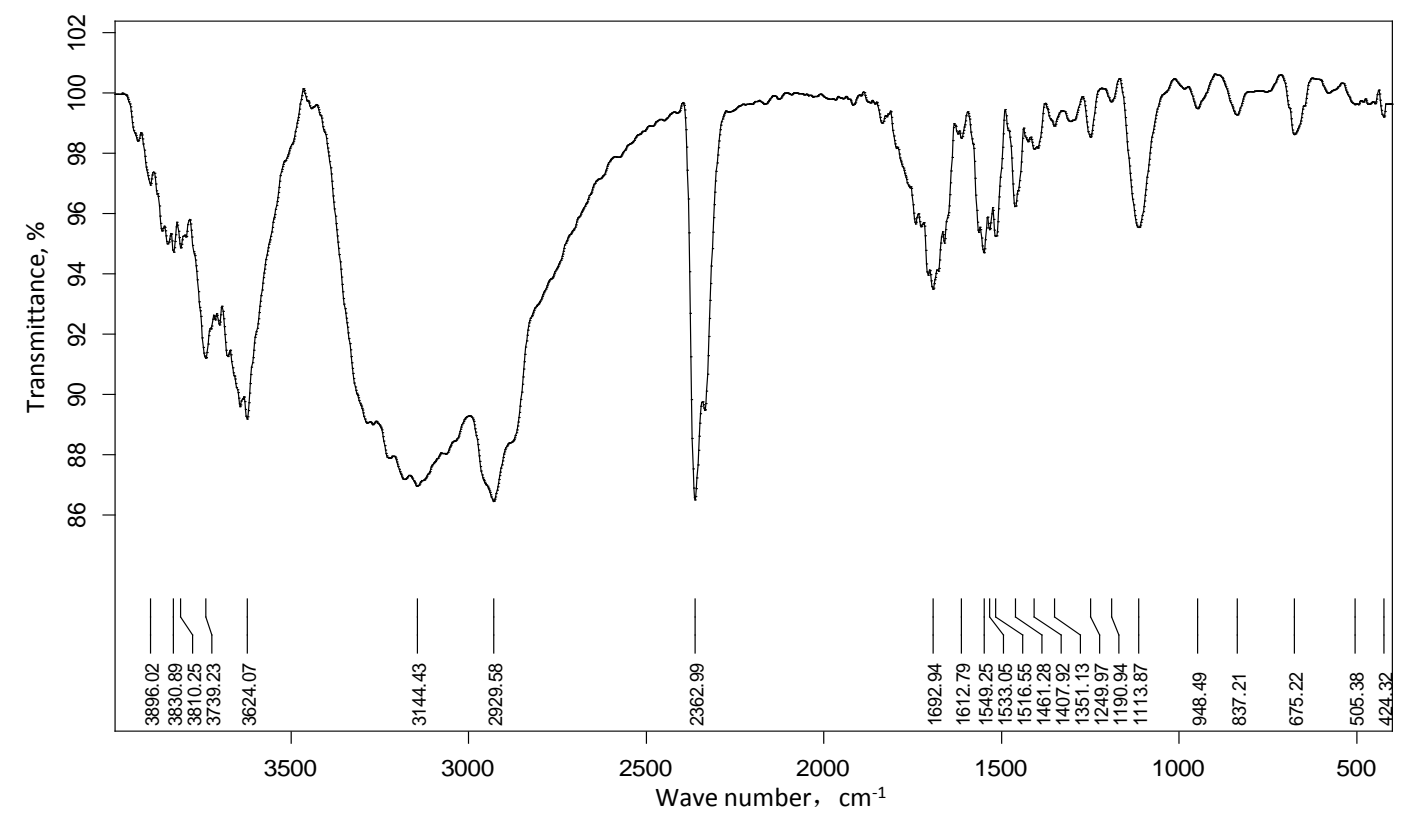

Figure 3. The infrared spectra of Lianhua S-PAM.

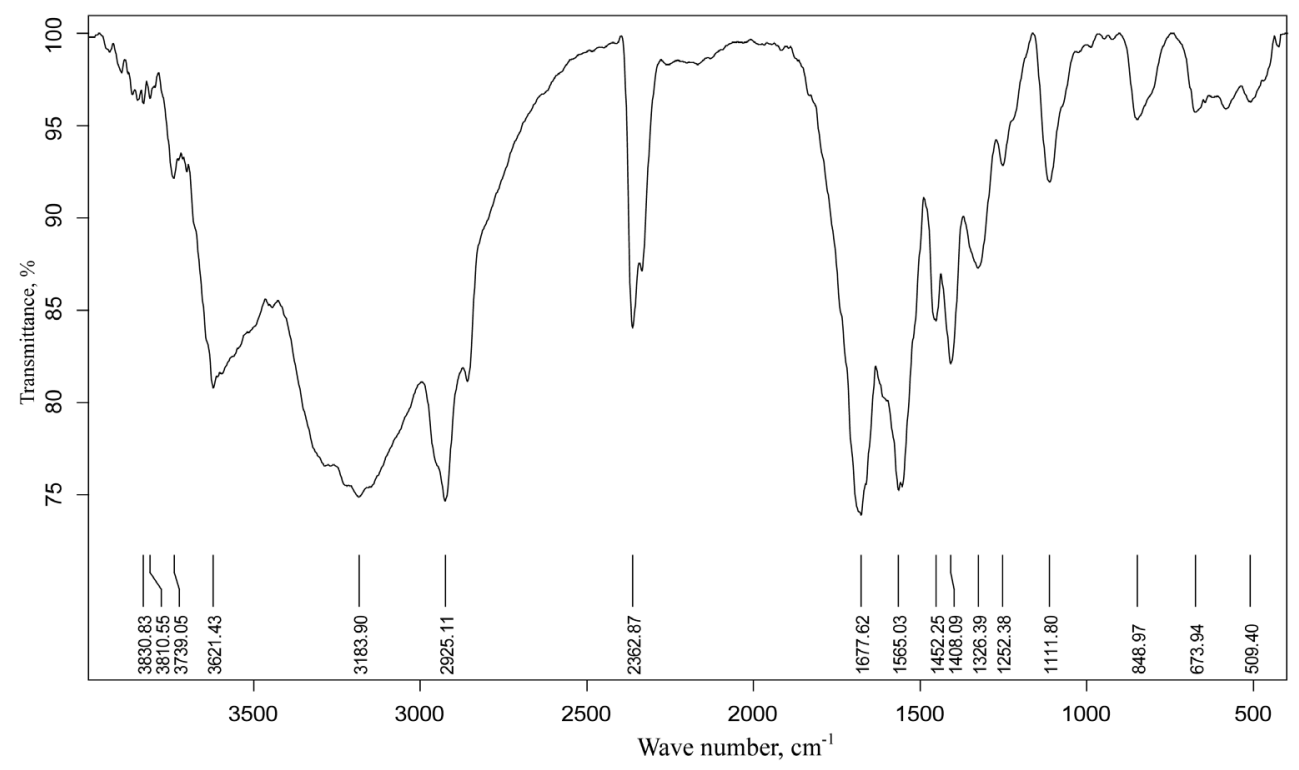

Figure 4. The infrared spectrum of Haibo S-PAM. 
Table 1. Primary determination results on anions.

\begin{tabular}{ccc}
\hline & Lianhua S-PAM & Haibo S-PAM \\
\hline $\mathrm{SO}_{4}^{2-}$ & Exclude & Include \\
$\mathrm{Cl}^{-}$ & Include & Include \\
\hline
\end{tabular}

Table 2. The analysis result on the infrared spectra of Lianhua S-PAM.

\begin{tabular}{ccc} 
No & Wave number range $\left(\mathrm{cm}^{-1}\right)$ & Functional groups \\
\hline 1 & Near 500 (intensive) & -C-I \\
2 & Near 690 & -CH=CH-(cis-) \\
3 & 890 - 820 (extremely weak) & Alkyl peroxide \\
4 & 970 - 960 (intensive) & -CH=CH-(trans-) \\
5 & 1150 - 1060 (extremely intensive) & Alkyl ethers \\
6 & 1220 - 1150 & Alkane sulfonate \\
7 & Near 1250 (intensive) & Cyclic ethers (epoxy compound) \\
8 & $1420-1330$ (intensive) 1610 - 1550 (intensive) & -COO- \\
9 & 1660 - 1480 (variable) & Ring-like conjugated compound \\
10 & 1667 - 833 & acylamino \\
11 & 1685 - 1665 & -CH=CH-CO- \\
12 & 2640 - 1630 & COOH oscillation in acylamino acid \\
13 & $2700-2560$ (wide and low) & P-OH \\
14 & 3300 - 3030 (extremely intesive), 1430 - 1390 (intensive) & Ammonium salt \\
\hline
\end{tabular}

Table 3. The analysis results on the infrared spectra of Haibo S-PAM.

\begin{tabular}{|c|c|c|}
\hline No & Wave number $\left(\mathrm{cm}^{-1}\right)$ & Functional groups \\
\hline 1 & Near 500 (intensive) & $-\mathrm{C}-\mathrm{I}$ \\
\hline 2 & Near 690 & $-\mathrm{CH}=\mathrm{CH}-($ cis -$)$ \\
\hline 3 & 890 - 820 (extremely weak) & Alkyl peroxide \\
\hline 4 & 1150 - 1060 (extremely weak) & Alkyl ethers \\
\hline 5 & $1250 \pm 5$ (intensive), near 1250 - 1200 (intensive) & $\left(\mathrm{CH}_{3}\right)_{3}-\mathrm{C}-\mathrm{R}$ \\
\hline 6 & 1340 - 1250 (intensive) & Secondary aromatic amines \\
\hline 7 & 1610 - 1550 (intensive) 1420 - 1300 (intensive) & $-\mathrm{COO}-$ \\
\hline 8 & $1465-1340$ & C-H alkanes with flexural vibration \\
\hline 9 & $1685-1665$ & $-\mathrm{CH}=\mathrm{CH}-\mathrm{CO}-$ \\
\hline 10 & 2440 - 2350 (medium) & $\mathrm{P}-\mathrm{H}$ \\
\hline 11 & $3000-2850$ & $\mathrm{CH}$ alkanes with stretching vibration \\
\hline 12 & 3030 - 2500 a series of continuous bands (weak) & Amino acid hydrochloride \\
\hline
\end{tabular}




\subsubsection{The Structure and Composition of S-PAM}

By means of chemical analysis, IR spectra analysis, as well as related data concerning S-PAM synthesis, the chemical structures of two S-PAM are indicated as follows:

The structure and compositions of Lianhua S-PAM:<smiles>[Y]NC(=O)C(CC(C)C(N)=O)CC(CC(CC(C)C)C(N)=O)C(=O)O</smiles>

where X: Alkyl ethers- $\left(\mathrm{CH}_{2} \mathrm{CH}_{2} \mathrm{O}\right)_{\mathrm{m}}-\mathrm{R}$, nononic

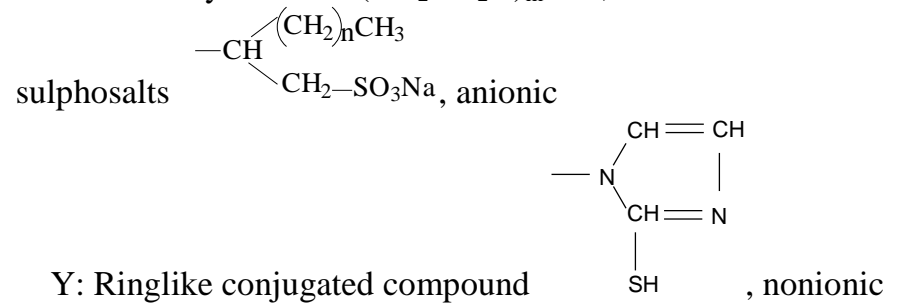

Plolyester $-\mathrm{CH}=\mathrm{CH}-\stackrel{\mathrm{O}}{\mathrm{C}}-\mathrm{O}-\left(\mathrm{CH}_{2}\right) \mathrm{nCH}_{3}$, nonionic

Carboxylates $-\mathrm{CH}=\mathrm{CH}-\stackrel{\mathrm{C}}{\mathrm{C}}-\mathrm{ONa}$, anionic

The structure and compositions of Haibo S-PAM:<smiles>[Y]NC(=O)C(CC(C)C(N)=O)CC(CC(CC(CC)C(N)=O)C(=O)O)C(=O)O[Y]</smiles>

where: $\mathrm{X}$ : Alkyl ethers $-\left(\mathrm{CH}_{2} \mathrm{CH}_{2} \mathrm{O}\right)_{\mathrm{m}}-\mathrm{R}$, nonionic

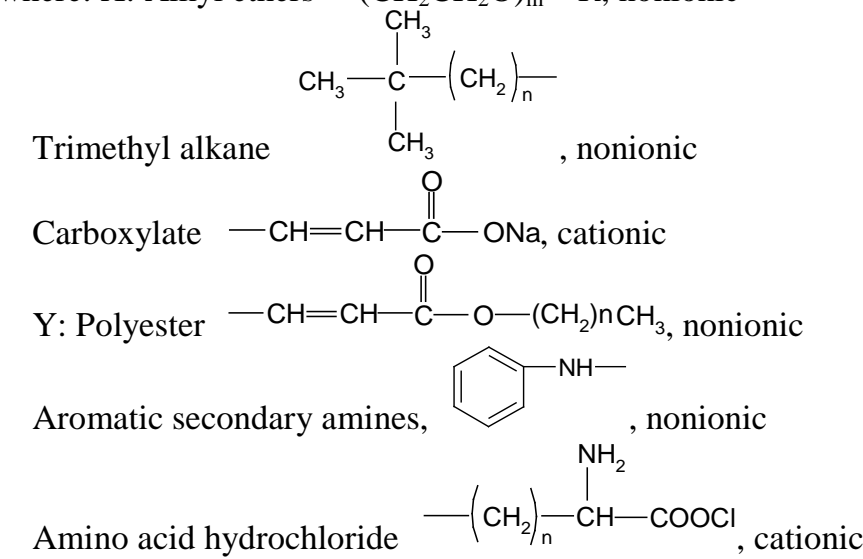

Analysis above showed that Lianhua S-PAM refining factory polymerized the nonionic and cationic surfactants, while that Haibo S-PAM polymerized the nonionic surfactants on the main chain of polyacrylamide. Moreover, the functional groups of the two S-PAM are different.

\section{Conclusion}

Analysis on the compositions of S-PAM implied that, Lianhua S-PAM polymerized the nonionic and cationic 
surfactants, while Haibo S-PAM polymerized the nonionic surfactants on the main chain of polyacrylamide. Moreover, the functional groups of the two polymer surfactants were different.

\section{References}

[1] Hu, B.Z. (1997) Production Engineering of Polymer Flooding. Petroleum Industry Press, Beijing, 70-72. (In Chinese)

[2] Ge, G.Z., Wang, J.Y. and Wang, Y.L. (2001) Polymer Flooding and Related Chemical Flooding Progress. Oilfield Chemistry, 18, 282-284. (In Chinese)

[3] Parrish, B., Breitenkamp, R.B. and Emrick, T. (2005) PEG- and Peptide-Grafted Aliphatic Polyesters by Click Chemistry. Journal of the American Chemical Society, 127, 7404-7410. http://dx.doi.org/10.1021/ja050310n

[4] Li, H.Y., Jérôme, R. and Lecomte, Ph. (2007) Combination of Ring-Opening Polymerization and "Click" Chemistry for the Synthesis of an Amphiphilic Tadpole-Shaped Poly( $\varepsilon$-Caprolactone) Grafted by PEO. Macromolecules, 40, 824-831. http://dx.doi.org/10.1021/ma062488f

[5] Zhang, J.Y., Zhou, Y.M., Liu, S.Y., et al. (2008) Polyion Complex Micelles Possessing Thermoresponsive Coronas and Their Covalent Core Stabilization via “Click" Chemistry. Macromolecules, 41, 1444-1454. http://dx.doi.org/10.1021/ma702199f

[6] Hizal, G., Tunca, U., Yagci, Y., et al. (2006) Anthracene-Maleimide-Based Diels-Alder “Click Chemistry” as a Novel Route to Graft Copolymers. Macromolecules, 39, 5330-5336. http://dx.doi.org/10.1021/ma060690c

[7] Zhao, J.R., Li, J.Y., Feng, Y., et al. (2007) A Novel Approach to Synthesis of Functional CPVC and CPE or Graft Copolymers- In Situ Chlorinating Graft. Polymers for Advanced Technologies, 18, 822-828. http://dx.doi.org/10.1002/pat.941

[8] Wang, Y.M., Wang, Y.J. and Lu, X.B. (2008) “Grafting-From” Polymerization for Uniformly Bulk Modification of Pre-Existing Polymer Materials via a Supercritical-Fluid Route. Polymer, 49, 474-480.

http://dx.doi.org/10.1016/j.polymer.2007.11.028 
Scientific Research Publishing (SCIRP) is one of the largest Open Access journal publishers. It is currently publishing more than 200 open access, online, peer-reviewed journals covering a wide range of academic disciplines. SCIRP serves the worldwide academic communities and contributes to the progress and application of science with its publication.

Other selected journals from SCIRP are listed as below. Submit your manuscript to us via either submit@scirp.org or Online Submission Portal.
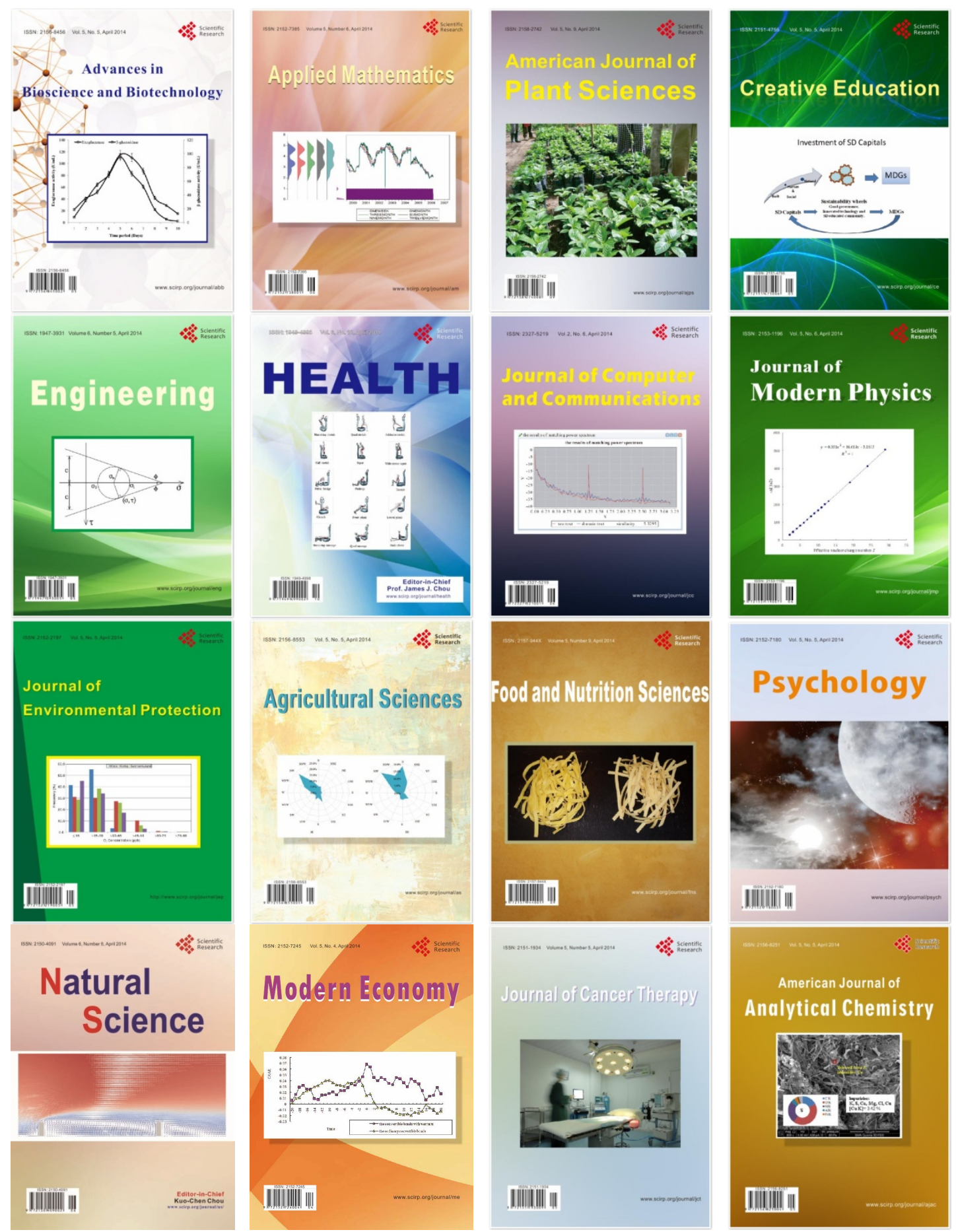